\title{
Emergence of oscillations in a mixed-mechanism phosphorylation system
}

\author{
Carsten Conradi; Maya Mincheva ${ }^{\dagger}$ and Anne Shiu ${ }^{\ddagger}$
}

January 28, 2019

\begin{abstract}
This work investigates the emergence of oscillations in one of the simplest cellular signaling networks exhibiting oscillations, namely, the dual-site phosphorylation and dephosphorylation network (futile cycle), in which the mechanism for phosphorylation is processive while the one for dephosphorylation is distributive (or vice-versa). The fact that this network yields oscillations was shown recently by Suwanmajo and Krishnan. Our results, which significantly extend their analyses, are as follows. First, in the three-dimensional space of total amounts, the border between systems with a stable versus unstable steady state is a surface defined by the vanishing of a single Hurwitz determinant. Second, this surface consists generically of simple Hopf bifurcations. Next, simulations suggest that when the steady state is unstable, oscillations are the norm. Finally, the emergence of oscillations via a Hopf bifurcation is enabled by the catalytic and association constants of the distributive part of the mechanism: if these rate constants satisfy two inequalities, then the system generically admits a Hopf bifurcation. Our proofs are enabled by the Routh-Hurwitz criterion, a Hopf-bifurcation criterion due to Yang, and a monomial parametrization of steady states.
\end{abstract}

Keywords: multisite phosphorylation, monomial parametrization, oscillation, Hopf bifurcation, Routh-Hurwitz criterion

\section{Introduction}

Oscillations have been observed experimentally in signaling networks formed by phosphorylation and dephosphorylation [20, 21, which suggests that these networks are involved in timekeeping and synchronization. Indeed, multisite phosphorylation is the main mechanism for establishing the 24-hour period in eukaryotic circadian clocks [30, 42]. Our motivating question, therefore, is, How do oscillations arise in phosphorylation networks?

We tackle this question for the network that, according to Suwanmajo and Krishnan, "could be the simplest enzymatic modification scheme that can intrinsically exhibit oscillation" [39, §3.1]. This network, in (1), is the mixed-mechanism (partially processive, partially

\footnotetext{
${ }^{*} \mathrm{HTW}$ Berlin

${ }^{\dagger}$ Northern Illinois University

†Texas A\&M University
} 
distributive) dual-site phosphorylation network (or mixed-mechanism network for short). Examples of networks that include both processive and distributive elements include the "processive model" of Aoki et al. [1, Table S2] and a model of ERK regulation via enzymes MEK and MKP3 [37, Fig. 2].

In the mixed-mechanism network, $S_{i}$ denotes a substrate with $i$ phosphate groups attached, and $K$ and $P$ are, respectively, a kinase and a phosphatase enzyme:

$$
\begin{aligned}
& S_{0}+K \underset{k_{2}}{\stackrel{k_{1}}{\rightleftarrows}} S_{0} K \stackrel{k_{3}}{\longrightarrow} S_{1} K \stackrel{k_{4}}{\longrightarrow} S_{2}+K \\
& S_{2}+P \underset{k_{6}}{\stackrel{k_{5}}{\rightleftarrows}} S_{2} P \stackrel{k_{7}}{\longrightarrow} S_{1}+P \underset{k_{9}}{\stackrel{k_{8}}{\rightleftarrows}} S_{1} P \stackrel{k_{10}}{\longrightarrow} S_{0}+P .
\end{aligned}
$$

When the kinase phosphorylates - that is, adds phosphate groups to - a substrate in the mixed-mechanism network (via the reactions labeled by $k_{1}$ to $k_{4}$ ), the kinase and substrate do not dissociate before both phosphate groups are added. Accordingly, the mechanism for phosphorylation is processive. In contrast, when the phosphatase dephosphorylates i.e., removes phosphate groups from - a substrate (via reactions $k_{5}$ to $k_{10}$ ), this mechanism is distributive: the phosphatase and substrate dissociate each time a phosphate group is removed. Accordingly, network (11) is said to have a mixed mechanism 1 .

The dynamical systems arising from the mixed-mechanism network live in a 9-dimensional space, but, due to three conservation laws, are essentially 6-dimensional. Specifically, the total amounts of kinase, phosphatase, and substrate - denoted by $K_{\text {tot }}$, $P_{\text {tot }}$, and $S_{\text {tot }}$, respectively - are conserved. For each choice of three such total amounts and each choice of positive rate constants $k_{i}$, there is a unique positive steady state [39]. One focus of our work is determining when such a steady state undergoes a Hopf bifurcation leading to oscillations (with any of the $k_{i}$ 's or total amounts as bifurcation parameter).

\subsection{Summary of main results}

How do oscillations of the mixed-mechanism network emerge, and how robust are they? These questions are the motivation for our work. Let us describe Suwanmajo and Krishnan's progress in this direction. They first found rate constants $k_{i}$ and total amounts, displayed in Table 1, that yield oscillations [39, Supplementary Information].

\begin{tabular}{|c|c|c|c|c|c|c|c|c|c|}
\hline$k_{1}$ & $k_{2}$ & $k_{3}$ & $k_{4}$ & $k_{5}$ & $k_{6}$ & $k_{7}$ & $k_{8}$ & $k_{9}$ & $k_{10}$ \\
\hline 1 & 1 & 1 & 1 & 100 & 1 & 0.9 & 3 & 1 & 100 \\
\hline
\end{tabular}

\begin{tabular}{|c|c|c|}
\hline$K_{\text {tot }}$ & $P_{\text {tot }}$ & $S_{\text {tot }}$ \\
\hline 17.5 & 5 & 40 \\
\hline
\end{tabular}

Table 1: Rate constants (left) and total amounts (right), from [39, Supplementary Information], which lead to oscillations in the mixed-mechanism network (1).

Next, they examined whether oscillations persist as $K_{\text {tot }}$ varies. What they found, summarized in Figure 1, is that oscillations persist when $K_{\text {tot }}$ is in the (approximate) interval $(13.03,29.23)$, and oscillations arise as the unique steady state undergoes a Hopf bifurcation.

\footnotetext{
${ }^{1}$ Network (1) is symmetric to the mixed-mechanism network in which phosphorylation is distributive (instead of processive) and dephosphorylation is processive (instead of distributive), so our results apply equally well to that network (cf. [39, networks 21-22]).
} 


\begin{tabular}{|lcccc}
$\begin{array}{c}\text { steady state is } \\
\text { locally stable }\end{array}$ & Hopf & $\begin{array}{c}\text { steady state is } \\
\text { unstable }\end{array}$ & Hopf & $\begin{array}{c}\text { steady state is } \\
\text { locally stable }\end{array}$ \\
\hline & $\approx 13.03$ & (oscillations) & $\approx 29.23$
\end{tabular}$K_{\text {tot }}$

Figure 1: Stability of the unique steady state of the mixed-mechanism network (1) as a function of $K_{\text {tot }}$, as analyzed by Suwanmajo and Krishnan [39, Fig. 4]. (The other total amounts, $P_{\text {tot }}$ and $S_{\text {tot }}$, and the rate constants $k_{i}$ are those in Table 1.) Oscillations were found when $K_{\text {tot }}$ is in the "unstable" interval [39].

Subsequently, Conradi and Shiu [7] found that when $P_{\text {tot }}$ also is allowed to vary, oscillations exist for larger values of $K_{\text {tot }}$ (e.g., $\left.K_{\text {tot }}=100\right)$. So, how exactly do oscillations depend on the three total amounts (or, equivalently, the initial conditions)? Concretely, our goal is to expand Figure 1 to encompass all possible perturbations to the initial conditions (i.e., the total amounts):

Question 1.1. Consider the mixed-mechanism network (1), with $k_{i}$ 's from Table 1 .

1. For which values of $\left(K_{\text {tot }}, P_{\text {tot }}, S_{\text {tot }}\right) \in \mathbb{R}_{>0}^{3}$ is the unique steady state unstable?

2. Whenever (by perturbing parameters or total amounts) a steady state switches from being locally stable to unstable, does this always give rise to a Hopf bifurcation?

The direct method for solving Question 1.1(1) is to solve the steady-state equations, and then apply the six-dimensional Routh-Hurwitz stability criterion. However, this approach is intractable: the resulting Hurwitz determinants are pages-long.

Accordingly, we take an algebraic shortcut. Namely, we find a parametrization of the set of steady states, and then use this for the input to Routh-Hurwitz. The result is somewhat surprising: each Hurwitz determinant except the last two (which are positive multiples of each other) is always positive. This yields our answer to Question 1.1(1): For every ODE system arising from the mixed-mechanism network (1), a (two-dimensional) surface in the three-dimensional space of total amounts defines the border between steady states that are stable and those that are unstable. (Our result even applies to many systems for which the $k_{i}$ 's are not those in Table 1, see Proposition 4.1.)

We can now translate Question 1.1(2) as follows: does the surface mentioned above consist of Hopf bifurcations? We prove, using a Hopf-bifurcation criterion stated in terms of Hurwitz determinants, due to Yang [43], that the answer, at least generically, is "yes": When the unique steady state of the mixed-mechanism network (1) switches from being stable to unstable, then, generically, it undergoes a Hopf bifurcation.

For general one-parameter ODE systems, there are two types of local bifurcations: saddle nodes (which require a zero eigenvalue of the Jacobian matrix) and Hopf bifurcations (which require a pair of pure imaginary eigenvalues of the Jacobian) [16]. We show that a saddle node bifurcation can not occur for any parameter values (see the proof of Proposition 4.1). Therefore, only Hopf bifurcations are possible for the mixed-mechanism system.

A second question we aim to answer is the following: 
Question 1.2. Consider the mixed-mechanism network (11). What conditions on the $k_{i}$ 's guarantee a Hopf-bifurcation for some (positive) values of the total concentrations?

As an answer to Question [1.2, we prove that the catalytic constants $\left(k_{7}\right.$ and $\left.k_{10}\right)$ and association constants $\left(k_{5}\right.$ and $k_{8}$ ) of the distributive part of the mechanism enable oscillations to emerge via a Hopf bifurcation. Specifically, under the simplifying assumption that all dissociation (backward-reaction) constants are equal $\left(k_{2}=k_{6}=k_{9}\right)$, if the rate constants satisfy two inequalities - lower bounds on $k_{10}$ and $k_{5} / k_{8}$ - then the system generically admits a Hopf bifurcation (Proposition 4.3 and Theorem 4.5). (As a comparison, for the fully distributive dual-site network described in Section 1.2 below, the catalytic constants alone enable bistability [5].) Finally, we encode the relevant inequalities in a procedure to generate many parameter values for which we expect oscillations (Procedure 5.1).

\subsection{Connection to related work}

Our work joins a growing number of works that harness steady-state parametrizations. Such results include criteria for when such parametrizations exist [26, 40] and methods for using them to determine whether a network is multistationary [25, 29, 32, 34]. Going further, steady-state parametrizations can also be used to find a witness to multistationarity or even the precise parameter regions that yield multistationarity [4, 5]. In this work, we use a steady-state parametrization in a novel way: to study oscillations via Hopf bifurcations. (Our approach is similar in spirit to using Clarke's convex parameters together with a Hopfbifurcation criterion [9, 11, 14, 18]).

As mentioned earlier, there has been much interest in the dynamics of phosphorylation systems [7]. The mixed-mechanism network (11) fits into the related literature as follows. The mixed network is a dual-site network situated between two extremes: the fully processive dual-site network - in which the phosphorylation and dephosphorylation mechanisms are both processive - and the fully distributive dual-site network. One might therefore expect the dynamics of the mixed-mechanism network to straddle those of the two networks. This is indeed the case. As summarized in Table 2, and reviewed in [7, fully processive networks are globally convergent to a unique steady state [6, 10, 35], while mixed-mechanism networks admit oscillations but not bistability [39], and fully distributive networks admit bistability [19] (and the question of oscillations is open [7]).

\begin{tabular}{lccc}
\hline Dual-site network & Oscillations? & Bistability? & Global convergence? \\
\hline Fully processive & No & No & Yes \\
Mixed-mechanism & Yes & No & No \\
Fully distributive & $($ Open $)$ & Yes & No \\
\hline
\end{tabular}

Table 2: Dual-site phosphorylation networks and their properties: whether they admit oscillations or bistability, and whether all trajectories converge to a unique steady state.

Finally, we revisit Suwanmajo and Krishnan's claim mentioned earlier that the mixedmechanism network is among the simplest enzymatic mechanisms with oscillations. In support of this claim, Tung proved that the simpler system obtained from the mixed-mechanism network by taking its (two-dimensional) Michaelis-Menten approximation, is not oscillatory 
[41. Moreover, Rao showed that this approximation is globally convergent to a unique steady state [36]. The validity of the Michaelis-Menten approximation for phosphorylation systems has been called into question [38], and what we know about the mixed-mechanism system concurs: this system is oscillatory, but its Michaelis-Menten approximation is not.

The outline of our work is as follows. Section 2 provides background on multisite phosphorylation, steady states, and Hopf bifurcations. Section 3 gives a monomial parametrization of the steady states of mixed-mechanism network. In Section 4, we prove our main results (described above). We use these results in Section 5 to give a procedure for generating rate constants admitting Hopf bifurcations. In Section 6, we present simulations that suggest that oscillations are the norm in the unstable-steady-state regime. Finally, we end with a Discussion in Section 7.

\section{Background}

In this section, we introduce the ODEs arising from the mixed-mechanism network, and recall two criteria: the Routh-Hurwitz criterion for steady-state stability and Yang's criterion for Hopf bifurcations.

\subsection{Differential equations of the mixed-mechanism network}

For the mixed-mechanism network (1), we let $x_{1}, x_{2}, \ldots, x_{9}$ denote the species concentrations in the order given in Table 3. The dynamical system (arising from mass-action kinetics) defined by the mixed-mechanism network (1) is given by the following ODEs:

$$
\begin{aligned}
& \dot{x}_{1}=-k_{1} x_{1} x_{2}+k_{2} x_{3}+k_{10} x_{9} \\
& \dot{x}_{2}=-k_{1} x_{1} x_{2}+k_{2} x_{3}+k_{4} x_{4} \\
& \dot{x}_{3}=k_{1} x_{1} x_{2}-\left(k_{2}+k_{3}\right) x_{3} \\
& \dot{x}_{4}=k_{3} x_{3}-k_{4} x_{4} \\
& \dot{x}_{5}=k_{4} x_{4}-k_{5} x_{5} x_{6}+k_{6} x_{7} \\
& \dot{x}_{6}=-k_{5} x_{5} x_{6}-k_{8} x_{8} x_{6}+\left(k_{6}+k_{7}\right) x_{7}+\left(k_{9}+k_{10}\right) x_{9} \\
& \dot{x}_{7}=k_{5} x_{5} x_{6}-\left(k_{6}+k_{7}\right) x_{7} \\
& \dot{x}_{8}=k_{7} x_{7}-k_{8} x_{6} x_{8}+k_{9} x_{9} \\
& \dot{x}_{9}=k_{8} x_{6} x_{8}-\left(k_{9}+k_{10}\right) x_{9} .
\end{aligned}
$$

\begin{tabular}{|c|c|c|c|c|c|c|c|c|}
\hline$x_{1}$ & $x_{2}$ & $x_{3}$ & $x_{4}$ & $x_{5}$ & $x_{6}$ & $x_{7}$ & $x_{8}$ & $x_{9}$ \\
\hline$S_{0}$ & $K$ & $S_{0} K$ & $S_{1} K$ & $S_{2}$ & $P$ & $S_{2} P$ & $S_{1}$ & $S_{1} P$ \\
\hline
\end{tabular}

Table 3: Assignment of variables to species for the mixed-mechanism network (1).

The conservation laws arise from the fact that the total amounts of free and bound enzyme or substrate remain constant. That is, as the dynamical system (2) progresses, the 
following three conservation values, denoted by $K_{\text {tot }}, P_{\text {tot }}, S_{\text {tot }} \in \mathbb{R}_{>0}$, remain constant:

$$
\begin{aligned}
K_{\text {tot }} & =x_{2}+x_{3}+x_{4}, \\
P_{\text {tot }} & =x_{6}+x_{7}+x_{9}, \\
S_{\text {tot }} & =x_{1}+x_{3}+x_{4}+x_{5}+x_{7}+x_{8}+x_{9} .
\end{aligned}
$$

Also, a trajectory $x(t)$ beginning in $\mathbb{R}_{\geq 0}^{9}$ remains in $\mathbb{R}_{\geq 0}^{9}$ for all positive time $t$, so it remains in a stoichiometric compatibility class, which we denote as follows:

$$
\mathcal{P}=\left\{x \in \mathbb{R}_{\geq 0}^{9} \mid \text { the conservation equations (3) hold }\right\} .
$$

\subsection{Stability of steady states and the Routh-Hurwitz criterion}

The dynamical system (2) arising from the mixed-mechanism network is an example of a reaction kinetics system. That is, the system of ODEs takes the following form:

$$
\frac{d x}{d t}=\Gamma \cdot R(x)=: g(x),
$$

where $\Gamma$ and $R$ are as follows. Letting $s$ denote the number of species and $r$ the number of reactions, $\Gamma$ is an $s \times r$ matrix whose $k$-th column is the reaction vector of the $k$-th reaction, i.e., it encodes the net change in each species that results when that reaction takes place. Also, $R: \mathbb{R}_{\geq 0}^{s} \rightarrow \mathbb{R}_{\geq 0}^{r}$ encodes the reaction rates of the $r$ reactions as functions of the $s$ species concentrations.

A steady state (respectively, positive steady state) of a reaction kinetics system is a nonnegative concentration vector $x^{*} \in \mathbb{R}_{>0}^{s}$ (respectively, $x^{*} \in \mathbb{R}_{>0}^{s}$ ) at which the ODEs (5) vanish: $g\left(x^{*}\right)=0$. Letting $S:=\operatorname{im}(\Gamma)$ denote the stoichiometric subspace, a steady state $x^{*}$ is nondegenerate if $\operatorname{Im}\left(\left.d g\left(x^{*}\right)\right|_{S}\right)=S$, where $d g\left(x^{*}\right)$ denotes the Jacobian matrix of $g$ at $x^{*}$.

A nondegenerate steady state is locally asymptotically stable if each of the $\sigma:=\operatorname{dim}(S)$ nonzero eigenvalues of $d g\left(x^{*}\right)$ has negative real part. Hence, a steady state is locally stable if and only if the characteristic polynomial of the Jacobian evaluated at the steady state has $\sigma$ roots with negative real part (the remaining roots will be 0 ).

To check whether a polynomial has only roots with negative real parts, we appeal to the Routh-Hurwitz criterion below [13].

Definition 2.1. The $i$-th Hurwitz matrix of a univariate polynomial $p(\lambda)=a_{0} \lambda^{n}+a_{1} \lambda^{n-1}+$ $\cdots+a_{n}$ is the following $i \times i$ matrix:

$$
H_{i}=\left(\begin{array}{ccccccc}
a_{1} & a_{0} & 0 & 0 & 0 & \cdots & 0 \\
a_{3} & a_{2} & a_{1} & a_{0} & 0 & \cdots & 0 \\
\vdots & \vdots & \vdots & \vdots & \vdots & & \vdots \\
a_{2 i-1} & a_{2 i-2} & a_{2 i-3} & a_{2 i-4} & a_{2 i-5} & \cdots & a_{i}
\end{array}\right),
$$

in which the $(k, l)$-th entry is $a_{2 k-l}$ as long as $0 \leq 2 k-l \leq n$, and 0 otherwise.

Proposition 2.2 (Routh-Hurwitz criterion). A polynomial $p(\lambda)=a_{0} \lambda^{n}+a_{1} \lambda^{n-1}+\cdots+a_{n}$ with $a_{0}>0$ has all roots with negative real part if and only if all $n$ of its Hurwitz matrices have positive determinant ( $\operatorname{det} H_{i}>0$ for all $i=1, \ldots, n$ ). 


\subsection{Hopf bifurcations and a criterion due to Yang}

A simple Hopf bifurcation is a bifurcation in which a single complex-conjugate pair of eigenvalues of the Jacobian matrix crosses the imaginary axis, while all other eigenvalues remain with negative real parts. Such a bifurcation, if it is supercritical, generates nearby oscillations or periodic orbits [27].

To detect simple Hopf bifurcations, we will use a criterion of Yang that characterizes Hopf bifurcations in terms of Hurwitz-matrix determinants (Proposition 2.3).

Setup for Yang's criterion. We consider an ODE system parametrized by $\mu \in \mathbb{R}$ :

$$
\dot{x}=g_{\mu}(x),
$$

where $x \in \mathbb{R}^{n}$, and $g_{\mu}(x)$ varies smoothly in $\mu$ and $x$. Assume that $x_{0} \in \mathbb{R}^{n}$ is a steady state of the system defined by $\mu_{0}$, that is, $g_{\mu_{0}}\left(x_{0}\right)=0$. Assume, furthermore, that we have a smooth curve of steady states:

$$
\mu \mapsto x(\mu)
$$

(that is, $g_{\mu}(x(\mu))=0$ for all $\mu$ ) and that $x\left(\mu_{0}\right)=x_{0}$. Denote the characteristic polynomial of the Jacobian matrix of $g_{\mu}$, evaluated at $x(\mu)$, as follows:

$$
p_{\mu}(\lambda):=\left.\operatorname{det}\left(\lambda I-\operatorname{Jac} g_{\mu}\right)\right|_{x=x(\mu)}=\lambda^{n}+a_{1}(\mu) \lambda^{n-1}+\cdots+a_{n}(\mu),
$$

and, for $i=1, \ldots, n$, let $H_{i}(\mu)$ denote the $i$-th Hurwitz matrix of $p_{\mu}(\lambda)$.

Proposition 2.3 (Yang's criterion [43]). Assume the above setup. Then, there is a simple Hopf bifurcation at $x_{0}$ with respect to $\mu$ if and only if the following hold:

(i) $a_{n}\left(\mu_{0}\right)>0$,

(ii) $\operatorname{det} H_{1}\left(\mu_{0}\right)>0$, $\operatorname{det} H_{2}\left(\mu_{0}\right)>0, \ldots$, det $H_{n-2}\left(\mu_{0}\right)>0$, and

(iii) $\operatorname{det} H_{n-1}\left(\mu_{0}\right)=0$ and $\left.\frac{d\left(\operatorname{det} H_{n-1}(\mu)\right)}{d \mu}\right|_{\mu=\mu_{0}} \neq 0$.

Remark 2.4. Liu [27] gave an earlier version of Yang's Hopf-bifurcation criterion (Proposition 2.3), using a variant of the Hurwitz matrices that differs from ours.

\section{Steady states of the mixed-mechanism network}

In this section, we recall that the mixed-mechanism network admits a unique steady state in each compatibility class (Proposition 3.1), and prove that the set of steady states admits a monomial parametrization (Theorem 3.2). We then use this parametrization to analyze the space of compatibility classes (Proposition 3.6). 


\subsection{Uniqueness of steady states}

Suwanmajo and Krishnan proved that, for every choice of positive rate constants and positive total amounts, the mixed-mechanism network does not admit multiple positive steady states [39, §A.2]. Additionally, there are no boundary steady states in any compatibility class $\mathcal{P}$, as in (4), and $\mathcal{P}$ is compact. Hence, via a standard application of the Brouwer fixed-point theorem (e.g., [33, Remark 3.9]), there is always a unique steady state:

Proposition 3.1 (Uniqueness of steady states). For any choice of positive rate constants $k_{i}$ and positive total amounts $K_{t o t}, P_{t o t}$, and $S_{t o t}$, the dynamical system (2) arising from the mixed-mechanism network has a unique steady state in $\mathcal{P}$, and it is a positive steady state.

Proposition 3.1 precludes the existence of multiple positive steady states, and hence the existence of a saddle node bifurcation. Thus, a Hopf bifurcation is the only other oneparameter bifurcation which may occur. Indeed, we will show that a Hopf bifurcation exists for some parameter values in Section 4 .

Also, Proposition 3.1 proves part of a conjecture that we posed [6]. The other half of the conjecture, however, posited that mixed-mechanism systems, like fully processive systems [6, 10], are globally convergent to the unique steady state. Suwanmajo and Krishnan demonstrated that this is false: the system can exhibit oscillatory behavior [39]!

This capacity for oscillations is the focus of this work, and our analysis will harness a monomial parametrization of the steady states. We turn to this topic now.

\subsection{A monomial parametrization of the steady states}

The steady states of the mixed-mechanism network can be parametrized by monomials (and thus is said to have "toric steady states" [33]):

Proposition 3.2 (Parametrization of the steady states). For every choice of rate constants $k_{i}>0$, the set of positive steady states of the mixed-mechanism system (2) is threedimensional and is the image of the following map $\chi=\chi_{k_{1}, \ldots, k_{10}}$ :

$$
\begin{aligned}
\chi: \mathbb{R}_{+}^{3} & \rightarrow \mathbb{R}_{+}^{9} \\
\left(x_{1}, x_{2}, x_{6}\right) & \mapsto\left(x_{1}, x_{2}, \ldots, x_{9}\right),
\end{aligned}
$$

given by

$$
\begin{aligned}
& x_{3}:=\frac{k_{1}}{k_{2}+k_{3}} x_{1} x_{2}, \quad x_{4}:=\frac{k_{1} k_{3}}{\left(k_{2}+k_{3}\right) k_{4}} x_{1} x_{2}, \quad x_{5}:=\frac{k_{1} k_{3}\left(k_{6}+k_{7}\right)}{\left(k_{2}+k_{3}\right) k_{5} k_{7}} \frac{x_{1} x_{2}}{x_{6}}, \\
& x_{7}:=\frac{k_{1} k_{3}}{\left(k_{2}+k_{3}\right) k_{7}} x_{1} x_{2}, \quad x_{8}:=\frac{k_{1} k_{3}\left(k_{9}+k_{10}\right)}{\left(k_{2}+k_{3}\right) k_{8} k_{10}} \frac{x_{1} x_{2}}{x_{6}}, \quad x_{9}:=\frac{k_{1} k_{3}}{\left(k_{2}+k_{3}\right) k_{10}} x_{1} x_{2} .
\end{aligned}
$$

Proof. It is straightforward to check that the image of $\chi$ is contained in the set of steady states: after substituting $\chi\left(x_{1}, x_{2}, x_{3}\right)$, the right-hand side of the mixed-mechanism network ODEs (2) vanishes. Conversely, let $x^{*}=\left(x_{1}, x_{2}, \ldots, x_{9}\right)$ be a positive steady state. The right-hand side of the ODEs (2) vanish at $x^{*}$, so, in the following order, we use $\dot{x}_{3}=0$ to solve for $x_{3}$ in terms of $x_{1}$ and $x_{2}$, use $\dot{x}_{4}=0$ to solve for $x_{4}$ via $x_{3}$ which was already 
obtained, use $\dot{x}_{1}=0$ to obtain $x_{9}$, use $\dot{x}_{9}=0$ to obtain $x_{8}$, use $\dot{x}_{8}=0$ to obtain $x_{7}$, and finally use $\dot{x}_{7}=0$ to obtain $x_{5}$. This yields precisely the parametrization (77), so $x^{*}$ is in the image of $\chi$.

Remark 3.3. The parametrization (7) appeared earlier in [7].

Remark 3.4. That we could achieve a steady-state parametrization was expected, due to Thomson and Gunawardena's rational parametrization theorem for multisite systems [40].

Remark 3.5. In the parametrization $\chi$ in Theorem 3.2 , we divide by $x_{6}$, so $\chi$ is technically not a monomial map. However, $\chi$ can be made monomial: we introduce $y:=\frac{x_{1}}{x_{6}}$, so that the parametrization accepts as input $\left(y, x_{2}, x_{6}\right)$, and then $x_{1}$ is replaced by $y x_{6}$.

\subsection{A parametrization of the compatibility classes}

Every compatibility class $\mathcal{P}$ of the mixed-mechanism network, by definition (4), is uniquely determined by a choice of total amounts $\left(K_{\text {tot }}, P_{\text {tot }}, S_{\text {tot }}\right) \in \mathbb{R}_{>0}^{3}$. Thus, we identify the set of compatibility classes with $\left\{\left(K_{\text {tot }}, P_{\text {tot }}, S_{\text {tot }}\right)\right\}=\mathbb{R}_{>0}^{3}$. We parametrize this set below (Proposition 3.6).

Let $\phi: \mathbb{R}_{>0}^{9} \rightarrow \mathbb{R}_{>0}^{3}$ denote the map sending a vector of concentrations to the corresponding total amounts $\left(K_{\text {tot }}, P_{\text {tot }}, S_{\text {tot }}\right)$, as in (3):

$$
\phi(x):=\left(x_{2}+x_{3}+x_{4}, x_{6}+x_{7}+x_{9}, x_{1}+x_{3}+x_{4}+x_{5}+x_{7}+x_{8}+x_{9}\right) \text {. }
$$

Each compatibility class $\mathcal{P}$ contains a unique positive steady state (Proposition 3.1), and the positive steady states are parametrized by $\chi$ from Theorem 3.2 , so the space of compatibility classes is parametrized as follows:

Proposition 3.6 (Parametrization of the compatibility classes). Identify every compatibility class $\mathcal{P}$ of the mixed-mechanism network (1), with the corresponding total amounts $\left(K_{\text {tot }}, P_{\text {tot }}, S_{\text {tot }}\right) \in \mathbb{R}_{>0}^{3}$. Then, for every choice of positive rate constants $k_{i}$, the following is a bijection that sends a vector $\left(x_{1}, x_{2}, x_{6}\right) \in \mathbb{R}_{>0}^{3}$ to the compatibility class in which the unique steady state is $\chi\left(x_{1}, x_{2}, x_{6}\right)$ :

$$
\phi \circ \chi: \mathbb{R}_{>0}^{3} \rightarrow \mathbb{R}_{>0}^{3}=\left\{\left(K_{\text {tot }}, P_{\text {tot }}, S_{\text {tot }}\right)\right\},
$$

where $\phi$ is as in (8) and $\chi$ is the steady-state parametrization (17). The map $\phi \circ \chi$ is given by

$$
\begin{aligned}
\left(x_{1}, x_{2}, x_{6}\right) \mapsto & \left(x_{2}+\frac{k_{1}}{k_{2}+k_{3}}\left(1+\frac{k_{3}}{k_{4}}\right) x_{1} x_{2}, \quad x_{6}+\frac{k_{1} k_{3}}{k_{2}+k_{3}}\left(\frac{1}{k_{7}}+\frac{1}{k_{10}}\right) x_{1} x_{2},\right. \\
& \left.x_{1}+\frac{k_{1} k_{3}}{k_{2}+k_{3}}\left[\left(\frac{1}{k_{3}}+\frac{1}{k_{4}}+\frac{1}{k_{7}}+\frac{1}{k_{10}}\right)+\frac{1}{x_{6}}\left(\frac{k_{6}+k_{7}}{k_{5} k_{7}}+\frac{k_{10}+k_{9}}{k_{10} k_{8}}\right)\right] x_{1} x_{2}\right),
\end{aligned}
$$

which becomes, when the rate constants are those in Table 1, the following:

$$
\left(x_{1}, x_{2}, x_{6}\right) \mapsto\left(x_{1} x_{2}+x_{2}, x_{6}+\frac{1009}{1800} x_{1} x_{2}, x_{1}+\frac{2809}{1800} x_{1} x_{2}+\frac{161}{900} \frac{x_{1} x_{2}}{x_{6}}\right) .
$$


Example 3.7. Consider the mixed-mechanism system with rate constants from Table1. To compute the unique steady state $x^{*}$ in the compatibility class given by $\left(K_{\text {tot }}, P_{\text {tot }}, S_{\text {tot }}\right)=$ $(17.5,5,40)$, we use Proposition 3.6. Namely, we know that $\phi \circ \chi\left(x_{1}^{*}, x_{2}^{*}, x_{6}^{*}\right)=(17.5,5,40)$, so we solve (using, e.g., Mathematica [22]) for the unique positive solution:

$$
\left(x_{1}^{*}, x_{2}^{*}, x_{6}^{*}\right) \approx(1.0134,8.6916,0.0624) .
$$

We obtain the remaining coordinates of $x^{*}$ using the parametrization $\chi$ in (7):

$$
\begin{aligned}
x^{*} & =\chi\left(x_{1}^{*}, x_{2}^{*}, x_{6}^{*}\right) \\
& \approx(1.0134,8.6916,4.4041,4.4041,1.4893,0.0624,4.8935,23.7512,0.0440) .
\end{aligned}
$$

\subsection{Steady states and Hopf bifurcations}

Our analysis of oscillations in the mixed-mechanism system is based on Hopf bifurcations. Hopf-bifurcation diagrams are displayed in Figure 2, where the total amounts are the bifurcation parameters (c.f. Figure 1 which is with respect to $K_{\text {tot }}$ ). Figure 2 suggests that, in the 3-dimensional space of total amounts, there is a surface of Hopf bifurcations. Indeed, we will see in the next section that this is the case (see Theorem 4.5 and Figure 3).

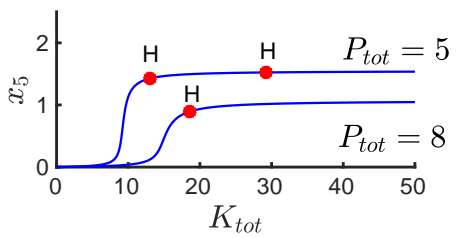

(a) Bif. parameter $K_{\text {tot }}$.

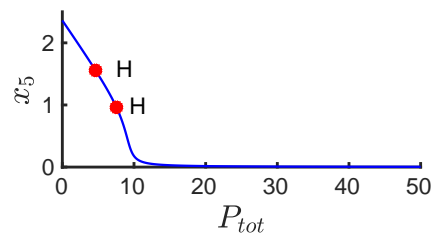

(b) Bif. parameter $P_{\text {tot }}$.

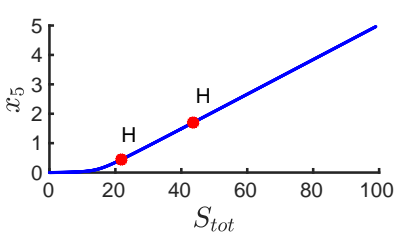

(c) Bif. parameter $S_{\text {tot }}$.

Figure 2: Numerical continuation of the unique positive steady state, in (10), when $\left(K_{\text {tot }}, P_{\text {tot }}, S_{\text {tot }}\right)=(17.5,5,40):$ (a) For $P_{\text {tot }}=5,8$ and $S_{\text {tot }}=40$, we observe $($ supercritical) Hopf bifurcations at $K_{\text {tot }} \approx 13.0296,29.2251\left(P_{\text {tot }}=5\right)$ and $K_{\text {tot }} \approx 18.5758$ $\left(P_{\text {tot }}=8\right)$. (b) For $K_{\text {tot }}=5$ and $S_{\text {tot }}=40$, we observe (supercritical) Hopf bifurcations at $P_{\text {tot }} \approx 4.6310$ and $P_{\text {tot }} \approx 7.5479$. (c) For $K_{\text {tot }}=17.5$ and $P_{\text {tot }}=5$, we observe (supercritical) Hopf bifurcations at $S_{\text {tot }} \approx 21.8213$ and $S_{\text {tot }} \approx 43.5944$. All figures in this work were made using Matcont [8].

\section{Hopf bifurcations in the mixed-mechanism system}

We saw in the previous section that the mixed-mechanism network yields a unique positive steady state in each compatibility class. Now we show that the compatibility classes with a stable steady state are separated from those with an unstable steady state by a single surface $\mathcal{H}$ (Proposition 4.1 and Theorem 4.2), and, under stronger hypotheses, crossing the surface $\mathcal{H}$ generically corresponds to undergoing a Hopf bifurcation (Theorem 4.5). (Recall that generically means that the exceptional set has zero measure. So, we will show that the subset of the surface corresponding to non-Hopf points has dimension at most 1.) 
To simplify computations, we assume that dissociation (backward-reaction) constants are equal: $k_{2}=k_{6}=k_{9}$. In chemistry, the forward reaction is usually more thermodynamically favorable than the backward reaction. Therefore, the rate constant of a forward reaction is much larger than the rate constant of the backward reaction [2]. We choose small values for the dissociation rate constants in Section 5, similar to what was done in [12].

Proposition 4.1. Consider the dynamical system (2) arising from the mixed-mechanism network and any positive rate constants for which $k_{2}=k_{6}=k_{9}$. Then:

1. Every compatibility class $\mathcal{P}$ contains a unique (positive) steady state $x^{*}$.

2. Exactly one of the following holds:

(a) The unique steady state $x^{*}$ in each compatibility class $\mathcal{P}$ is locally asymptotically stable.

(b) In the space of total amounts $\left\{\left(K_{\text {tot }}, P_{\text {tot }}, S_{\text {tot }}\right)\right\}=\mathbb{R}_{>0}^{3}$, which we identify with the space of compatibility classes $\mathcal{P}$, a surface $\mathcal{H}$ defines the border between those $\mathcal{P}$ whose unique steady state $x^{*}$ is locally asymptotically stable and those $\mathcal{P}$ for which $x^{*}$ is unstable.

Proof. Item 1 follows from Proposition 3.1.

For item 2 , let $J$ denote the Jacobian matrix of the mixed-mechanism system (2), with equal dissociation constants: $k_{2}=k_{6}=k_{9}=: k_{b}$, evaluated at the parametrized steady state $\chi\left(x_{1}, x_{2}, x_{6}\right)$, from (7). The characteristic polynomial of $J$ is:

$$
p(\lambda):=\operatorname{det}(\lambda I-J)=\lambda^{3}\left(\lambda^{6}+b_{1} \lambda^{5}+b_{2} \lambda^{4}+\cdots+b_{6}\right),
$$

where the coefficients $b_{i}$ (displayed below) are rational functions in $x_{1}, x_{2}, x_{6}$ and the $k_{i}$ 's. To streamline reading we only give the complete numerator of $b_{6}$ and $b_{1}$. The full coefficients can be found in the Mathematica file mixed_coeffs_charpoly_kb.nb2

$$
\begin{aligned}
& \operatorname{numerator}\left(b_{6}\right)=k_{1}^{2} k_{3}^{2} k_{4}\left(k_{10}+k_{7}\right)\left(k_{10} k_{5} k_{7}+k_{5} k_{7} k_{b}+k_{10} k_{8}\left(k_{7}+k_{b}\right)\right) x_{1} x_{2}^{2} \\
& +k_{1} k_{10} k_{3} k_{4} k_{7}\left(k_{3}+k_{b}\right)\left(k_{10} k_{5} k_{7}+k_{5} k_{7} k_{b}+k_{10} k_{8}\left(k_{7}+k_{b}\right)\right) x_{2} x_{6} \\
& +k_{10}^{2} k_{4} k_{5} k_{7}^{2} k_{8}\left(k_{3}+k_{b}\right)^{2} x_{6}^{2}+k_{1} k_{10}^{2}\left(k_{3}+k_{4}\right) k_{5} k_{7}^{2} k_{8}\left(k_{3}+k_{b}\right) x_{1} x_{6}^{2} \\
& +k_{1} k_{10} k_{5} k_{7}\left(k_{10} k_{4} k_{7}+k_{3} k_{4} k_{7}+k_{10} k_{3}\left(k_{4}+k_{7}\right)\right) k_{8}\left(k_{3}+k_{b}\right) x_{2} x_{6}^{2} \\
& +k_{1} k_{10} k_{3} k_{4} k_{7}\left(k_{10}+k_{b}\right)\left(k_{3}+k_{b}\right)\left(k_{7}+k_{b}\right) x_{2} x_{6}+\ldots \\
& \text { numerator }\left(b_{4}\right)=k_{1} k_{3} k_{4}\left(k_{10}+k_{7}\right)\left(k_{10}+k_{b}\right)\left(k_{3}+k_{b}\right)\left(k_{7}+k_{b}\right) x_{1} x_{2}+\ldots \\
& \operatorname{numerator}\left(b_{3}\right)=\ldots+k_{1}^{2} k_{3}\left(k_{10}^{2}\left(k_{7}+k_{b}\right)+k_{7} k_{b}\left(k_{3}+k_{4}+k_{7}+k_{b}\right)\right. \\
& \left.+k_{10}\left(\left(k_{7}+k_{b}\right)^{2}+k_{3}\left(2 k_{7}+k_{b}\right)+k_{4}\left(2 k_{7}+k_{b}\right)\right)\right) x_{1}^{2} x_{2}+\ldots \\
& \operatorname{numerator}\left(b_{2}\right)=\ldots+k_{1}^{2} k_{3}\left(k_{7} k_{b}+k_{10}\left(2 k_{7}+k_{b}\right)\right) x_{1}^{2} x_{2}+\ldots \\
& \text { numerator }\left(b_{1}\right)=k_{1} k_{3}\left(k_{7} k_{b}+k_{10}\left(2 k_{7}+k_{b}\right)\right) x_{1} x_{2}+k_{10} k_{7}\left(k_{3}+k_{b}\right)\left(k_{10}+k_{3}+k_{4}+k_{7}+3 k_{b}\right) x_{6} \\
& +k_{1} k_{10} k_{7}\left(k_{3}+k_{b}\right) x_{1} x_{6}+k_{1} k_{10} k_{7}\left(k_{3}+k_{b}\right) x_{2} x_{6}+k_{10} k_{7}\left(k_{5}+k_{8}\right)\left(k_{3}+k_{b}\right) x_{6}^{2}
\end{aligned}
$$

\footnotetext{
${ }^{2}$ This file and others mentioned below are in the Supporting Information; see Appendix A
} 
And for the denominators:

$$
\begin{aligned}
\text { denominator }\left(b_{6}\right) & =k_{10}\left(k_{b}+k_{3}\right) k_{7} \\
\text { denominator }\left(b_{i}\right) & =k_{10}\left(k_{b}+k_{3}\right) k_{7} x_{6}, \quad \text { for } i=2,3,4,5 .
\end{aligned}
$$

As $x_{1}, x_{2}, x_{6}$ and the $k_{i}$ are positive, thus $b_{1}, b_{2}, \ldots, b_{6}>0$ (in the aforementioned Mathematica file, we checked the above numerators are sums of only positive monomials).

Recall that, due to the 3 conservation laws (3), the Jacobian matrix has rank 6, not 9. Accordingly, the relevant Hurwitz matrix, namely, for $p(\lambda) / \lambda^{3}$, is as follows:

$$
\left(\begin{array}{cccccc}
b_{1} & 1 & 0 & 0 & 0 & 0 \\
b_{3} & b_{2} & b_{1} & 1 & 0 & 0 \\
b_{5} & b_{4} & b_{3} & b_{2} & b_{1} & 1 \\
0 & b_{6} & b_{5} & b_{4} & b_{3} & b_{2} \\
0 & 0 & 0 & b_{6} & b_{5} & b_{4} \\
0 & 0 & 0 & 0 & 0 & b_{6}
\end{array}\right)
$$

Consider the Hurwitz determinants. First det $H_{1}=b_{1}>0$. The next 3 Hurwitz determinants are also positive:

$$
\begin{aligned}
\text { numerator }\left(\operatorname{det} H_{2}\right)= & k_{1}^{3} k_{3}^{2}\left(k_{7} k_{b}+k_{10}\left(2 k_{7}+k_{b}\right)\right)^{2} x_{1}^{3} x_{2}^{2} \\
& +k_{1}^{3} k_{10} k_{3} k_{7}\left(k_{3}+k_{b}\right)\left(k_{7} k_{b}+k_{10}\left(2 k_{7}+k_{b}\right)\right) x_{1}^{3} x_{2} x_{6}+\ldots \\
\text { numerator }\left(\operatorname{det} H_{3}\right)= & k_{1}^{5} k_{3}^{3}\left(k_{10} k_{5} k_{7}+k_{5} k_{7} k_{b}+k_{10} k_{8}\left(k_{7}+k_{b}\right)\right)\left(k_{7} k_{b}+k_{10}\left(2 k_{7}+k_{b}\right)\right)^{2} x_{1}^{5} x_{2}^{3} x_{6}+\ldots \\
\text { numerator }\left(\operatorname{det} H_{4}\right)= & k_{1}^{7} k_{3}^{4}\left(k_{10} k_{5} k_{7}+k_{5} k_{7} k_{b}+k_{10} k_{8}\left(k_{7}+k_{b}\right)\right)\left(k_{7} k_{b}+k_{10}\left(2 k_{7}+k_{b}\right)\right)^{2} \\
& \left(k_{5} k_{7}\left(k_{3}+k_{4}+k_{7}\right) k_{b}+k_{10}^{2} k_{8}\left(k_{7}+k_{b}\right)+\right. \\
& \left.k_{10}\left(k_{3}+k_{4}+k_{7}\right)\left(k_{5} k_{7}+k_{8}\left(k_{7}+k_{b}\right)\right)\right) x_{1}^{7} x_{2}^{4} x_{6}^{2}+\ldots
\end{aligned}
$$

where the denominators, which are positive, are, respectively:

$$
\begin{aligned}
\text { denominator }\left(\operatorname{det} H_{2}\right) & =k_{10}^{2} k_{7}^{2}\left(k_{b}+k_{3}\right)^{2} x_{6}^{2} \\
\text { denominator }\left(\operatorname{det} H_{3}\right) & =k_{10}^{3} k_{7}^{3}\left(k_{b}+k_{3}\right)^{3} x_{6}^{3} \\
\text { denominator }\left(\operatorname{det} H_{4}\right) & =k_{10}^{4} k_{7}^{4}\left(k_{b}+k_{3}\right)^{4} x_{6}^{4}
\end{aligned}
$$

(We display only the leading terms of the polynomials; the complete polynomials together with an algorithmic verification of positivity are in mixed_Hi.nb.) The final Hurwitz determinant is det $H_{6}=\left(b_{6}\right)\left(\operatorname{det} H_{5}\right)$, and we saw that $b_{6}>0$. So, by the Routh-Hurwitz criterion (Proposition 2.2), the steady state $\chi\left(x_{1}, x_{2}, x_{6}\right)$ is locally stable if and only if $\operatorname{det} H_{5}>0$.

Hence, the surface $\mathcal{H}$ that delineates the boundary between compatibility classes with stable steady states vs. those with unstable steady states is defined by $\operatorname{det} H_{5} \circ(\phi \circ \chi)^{-1}=0$, where $\phi \circ \chi$ is the parametrization of compatibility classes from Proposition 3.6. If $\mathcal{H}$ intersects the positive orthant $\mathbb{R}_{>0}^{3}$, then case (b) of the proposition holds. Otherwise, if $\mathcal{H} \cap \mathbb{R}_{>0}^{3}=\emptyset$, then we claim that we are in case (a). To show this, we need to verify that $\operatorname{det} H_{5}\left(x_{1}, x_{2}, x_{6}\right)>0$ for some $\left(x_{1}, x_{2}, x_{6}\right) \in \mathbb{R}_{>0}^{3}$. The denominator of $\operatorname{det} H_{5}\left(x_{1}, x_{2}, x_{6}\right)$ is strictly positive:

$$
\text { denominator }\left(\operatorname{det} H_{5}\right)=k_{10}^{5} k_{7}^{5}\left(k_{3}+k_{b}\right)^{5} x_{6}^{5} \text {. }
$$


So we need only show that the numerator of $\operatorname{det} H_{5}\left(x_{1}, x_{2}, x_{6}\right)$ is strictly positive for some $\left(x_{1}, x_{2}, x_{6}\right) \in \mathbb{R}_{>0}^{3}$.

To this end, we view this numerator as a polynomial in $x_{1}$ (so the coefficients are rational functions of $x_{2}, x_{6}$, and the $k_{i}$ 's):

$$
\begin{aligned}
\text { numerator }\left(\operatorname{det} H_{5}\right)= & x_{1}^{9} x_{2}^{4}\left(\frac{k_{10} k_{7} x_{6}\left(k_{3}+k b\right)}{k_{3}\left(k_{10}\left(2 k_{7}+k b\right)+k_{7} k b\right)}+x_{2}\right) \\
& {\left[k_{8} x_{6}\left(\alpha_{01}+\alpha_{10} \frac{k_{5}}{k_{8}}\right)+k_{8}^{2} x_{6}^{2}\left(\alpha_{02}+\alpha_{11} \frac{k_{5}}{k_{8}}+\alpha_{20}\left(\frac{k_{5}}{k_{8}}\right)^{2}\right)+\right.} \\
& \left.k_{8}^{3} x_{6}^{3}\left(\alpha_{03}+\alpha_{12} \frac{k_{5}}{k_{8}}+\alpha_{21}\left(\frac{k_{5}}{k_{8}}\right)^{2}+\alpha_{30}\left(\frac{k_{5}}{k_{8}}\right)^{3}\right)\right]+ \text { lower degree terms in } x_{1},
\end{aligned}
$$

where the coefficients $\alpha_{i j}$ are sums of (many) positive monomials and are given in the file mixed_analyis_H5N_x1_LT.nb. Therefore (for fixed $x_{2}$ and $x_{6}$ ) when $x_{1}$ is sufficiently large, the expression (12) is positive, as desired.

The proof of Proposition 4.1 focused on the surface $\mathcal{H}$ defined by the equation det $H_{5} \circ$ $(\phi \circ \chi)^{-1}=0$. This surface sometimes meets the positive orthant $\mathbb{R}_{>0}^{3}$, and indeed we show that this is the case when certain relationships hold among the rate constants.

Theorem 4.2. Consider the dynamical system (21) arising from the mixed-mechanism network. Assume the positive rate constants satisfy $k_{2}=k_{6}=k_{9}$ and the following inequality:

$$
k_{10} k_{3} k_{4}-\left(k_{3}+k_{4}\right)\left(k_{3}+k_{7}\right)\left(k_{4}+k_{7}\right)>0 .
$$

If $k_{5} / k_{8}$ is sufficiently large, then there is a compatibility class $\mathcal{P}$ whose unique steady state $x^{*}$ is unstable.

Proof. Assume that the rate constants satisfy $k_{2}=k_{6}=k_{9}=: k_{b}$ and (13). By the proof of Proposition 4.1, a steady state $\chi\left(x_{1}, x_{2}, x_{6}\right)$ of the mixed-mechanism system (2) is locally stable if and only if det $H_{5}\left(x_{1}, x_{2}, x_{6}\right)>0$. We also saw in that proof that the denominator of det $H_{5}\left(x_{1}, x_{2}, x_{6}\right)$ is strictly positive for all $\left(x_{1}, x_{2}, x_{6}\right) \in \mathbb{R}_{>0}^{3}$. So, by Proposition 2.2 , it suffices to show that if $k_{5} / k_{8}$ is sufficiently large, then there exists $\left(x_{1}^{*}, x_{2}^{*}, x_{6}^{*}\right) \in \mathbb{R}_{>0}^{3}$ such that the numerator of det $H_{5}\left(x_{1}^{*}, x_{2}^{*}, x_{6}^{*}\right)$ is strictly negative: this would show that the steady state $x^{*}:=\chi\left(x_{1}^{*}, x_{2}^{*}, x_{6}^{*}\right)$ is unstable.

To this end, view the numerator of $\operatorname{det} H_{5}$ as a polynomial in $x_{2}$ with coefficients in $x_{1}, x_{6}$, and the $k_{i}$ 's. It is a degree-9 polynomial in $x_{2}$ of the following form (see the file mixed_analysis_H5N_x2_LT.nb):

numerator $\left(\operatorname{det} H_{5}\right)=k_{1}^{9}\left(\alpha_{0} x_{6}^{3}+\alpha_{1} x_{6}^{2}+\alpha_{2} x_{6}+\alpha_{3}\right)\left(x_{1}^{5}+\frac{k_{10} k_{7}\left(k_{3}+k_{b}\right)}{k_{3}\left(k_{10}\left(2 k_{7}+k_{b}\right)+k_{7} k_{b}\right)} x_{1}^{4} x_{6}\right) x_{2}^{9}$

+ lower degree terms ,

where $\alpha_{0}, \ldots, \alpha_{3}$ are rational functions in $k_{b}, k_{3}, k_{4}, k_{5}, k_{7}, k_{8}, k_{10}$. These functions $\alpha_{i}$ are given in mixed_analysis_H5N_x2_LT.nb. 
We now analyze $\alpha_{0}$, which has the following form (see mixed_analysis_H5N_x2_LT.nb):

$$
\alpha_{0}=k_{8}^{3}\left(\beta_{0}\left(\frac{k_{5}}{k_{8}}\right)^{3}+\beta_{1}\left(\frac{k_{5}}{k_{8}}\right)^{2}+\beta_{2}\left(\frac{k_{5}}{k_{8}}\right)+\beta_{3}\right),
$$

where each coefficient $\beta_{i}$ is a rational function in $k_{b}, k_{3}, k_{4}, k_{7}, k_{10}$ (and hence does not depend on $k_{1}, k_{5}$, or $k_{8}$ ). In particular, $\beta_{0}$ is the following polynomial:

$$
\beta_{0}=-k_{1}^{9} k_{3}^{5} k_{7}^{3}\left(k_{10} k_{3} k_{4}-\left(k_{3}+k_{4}\right)\left(k_{3}+k_{7}\right)\left(k_{4}+k_{7}\right)\right)\left(k_{10}+k_{b}\right)^{3}\left(k_{7} k_{b}+k_{10}\left(2 k_{7}+k_{b}\right)\right)^{2} .
$$

It follows that $\beta_{0}<0$ when inequality (13) holds.

Thus, when (13) holds, then, by equation (15), the inequality $\alpha_{0}<0$ holds for $k_{5} / k_{8}$ sufficiently large. In this case, the cubic polynomial in $x_{6}$ appearing in (14), and hence also the coefficient of $x_{2}^{9}$ in the numerator of $\operatorname{det} H_{5}$, will be negative for $x_{6}$ sufficiently large. Hence, if we choose $x_{1}:=1$ (or any positive value) and $x_{6}$ and $x_{2}$ sufficiently large, then the numerator of det $H_{5}$ will be negative.

In the remainder of this section, we focus on the question of whether the surface $\mathcal{H}$ consists of (at least generically) Hopf bifurcations. If so, this would imply that whenever a steady state of the mixed-mechanism network switches from stable to unstable, we expect it to undergo a Hopf bifurcation leading to oscillations. We begin our analyses of Hopf bifurcations by giving a criterion for such bifurcations.

Proposition 4.3. Consider the dynamical system (2) arising from the mixed-mechanism network and any positive rate constants with $k_{2}=k_{6}=k_{9}$ and $k_{10} k_{3} k_{4}-\left(k_{3}+k_{4}\right)\left(k_{3}+\right.$ $\left.k_{7}\right)\left(k_{4}+k_{7}\right)>0$. Then there exists $\left(x_{1}^{*}, x_{2}^{*}, x_{6}^{*}\right) \in \mathbb{R}_{>0}^{3}$ such that $\operatorname{det} H_{5}\left(x_{1}^{*}, x_{2}^{*}, x_{6}^{*}\right)=0$ (in other words, $\phi \circ \chi\left(x_{1}^{*}, x_{2}^{*}, x_{6}^{*}\right)$ is on $\left.\mathcal{H}\right)$. Moreover, for such a vector $\left(x_{1}^{*}, x_{2}^{*}, x_{6}^{*}\right)$, the system undergoes a Hopf bifurcation with respect to $x_{2}$ at the steady state $\chi\left(x_{1}^{*}, x_{2}^{*}, x_{6}^{*}\right)$ if and only if the following inequality holds:

$$
\left.\frac{d\left(\text { numerator }\left.\left(\operatorname{det} H_{5}\right)\right|_{x_{1}=x_{1}^{*}, x_{6}=x_{6}^{*}}\right)}{d x_{2}}\right|_{x_{2}=x_{2}^{*}} \neq 0 .
$$

Proof. Fix positive rate constants for which $k_{2}=k_{6}=k_{9}$ and $k_{10} k_{3} k_{4}-\left(k_{3}+k_{4}\right)\left(k_{3}+k_{7}\right)\left(k_{4}+\right.$ $\left.k_{7}\right)>0$. By the proofs of Proposition 4.1 and Theorem 4.2 , the function $\operatorname{det} H_{5}: \mathbb{R}_{>0}^{3} \rightarrow \mathbb{R}$ takes both positive and negative values. So, as $\operatorname{det} H_{5}$ is continuous, $\operatorname{det} H_{5}\left(x_{1}^{*}, x_{2}^{*}, x_{6}^{*}\right)=0$ for some $\left(x_{1}^{*}, x_{2}^{*}, x_{6}^{*}\right) \in \mathbb{R}_{>0}^{3}$ (by the intermediate-value theorem).

Assume det $H_{5}\left(x_{1}^{*}, x_{2}^{*}, x_{6}^{*}\right)=0$. To see whether the steady state $\chi\left(x_{1}^{*}, x_{2}^{*}, x_{6}^{*}\right)$ is a Hopf bifurcation with respect to the parameter $\mu=x_{2}$, where the curve of steady states is $x(\mu)=$ $\chi\left(x_{1}^{*}, \mu, x_{6}^{*}\right)$ and $\mu_{0}=x_{2}^{*}$, we use Proposition 2.3 (Yang's criterion). Parts (i) and (ii) of that criterion hold for any steady state $\chi\left(x_{1}^{*}, x_{2}^{*}, x_{6}^{*}\right)$, because $b_{6}=b_{6}\left(x_{1}^{*}, x_{2}^{*}, x_{6}^{*}\right)>0$, by (11), and also $\operatorname{det} H_{i}=\operatorname{det} H_{i}\left(x_{1}^{*}, x_{2}^{*}, x_{6}^{*}\right)>0$ for $i=1,2,3,4$ (from the proof of Proposition 4.1). Recall from the proof of Proposition 4.1 that the denominator of $\operatorname{det} H_{5}$ is strictly positive and does not depend on $x_{2}$; thus, we can focus on the numerator of $H_{5}$. So, by Proposition 2.3 , $\chi\left(x_{1}^{*}, x_{2}^{*}, x_{6}^{*}\right)$ is a Hopf bifurcation with respect $x_{2}$ if and only if (16) holds. 
Remark 4.4. Given rate constants $k_{i}$ as in Proposition 4.3 for which there is a Hopf bifurcation, we can perturb slightly the rate constants involved in (13) (while maintaining the equality $k_{2}=k_{6}=k_{9}$ ) and preserve the existence of a Hopf bifurcation. Indeed, this assertion follows from Proposition 4.3 (inequality (16) is maintained under small perturbations of the $x_{i}$ 's), the fact that simple roots of a polynomial depend continuously - in fact, infinitely differentiably - on the coefficients [28], and the fact that the inequality (13) defines a (relatively) open set in the parameter space of the $k_{i}$ 's.

Under the hypotheses of Proposition 4.3, we expect that inequality (16) holds generically on $\mathcal{H}$. We will confirm this when the rate constants are those in Table 1 (Theorem 4.5).

The proof of Theorem 4.5 makes use of discriminants, which we now review. Consider a degree- $n$, univariate polynomial $f=c_{n} x^{n}+c_{n-1} x^{n-1}+\cdots+c_{0}$ with coefficients $c_{i} \in \mathbb{C}$. A multiple root of $f$ is some $x^{*} \in \mathbb{C}$ for which $\left(x-x^{*}\right)^{2}$ divides $f$ or equivalently $f\left(x^{*}\right)=$ $f^{\prime}\left(x^{*}\right)=0$. It is well-known that $f$ has a multiple root in $\mathbb{C}$ if and only if a certain multivariate polynomial in the $c_{i}$ 's, the discriminant, vanishes [15]. For instance, the discriminant of the quadratic polynomial $a x^{2}+b x+c$ is the familiar expression $b^{2}-4 a c$.

Theorem 4.5 (Hopf bifurcations of the mixed-mechanism network). Consider the dynamical system (2) arising from the mixed-mechanism network and rate constants in Table 1. Let $\mathcal{H}$ denote the surface, from Proposition 4.1, that defines the border between those $\mathcal{P}$ whose unique steady state $x^{*}$ is locally stable and those $\mathcal{P}$ for which $x^{*}$ is unstable. Then $\mathcal{H}$ consists generically of compatibility classes $\mathcal{P}$ whose unique steady state $x^{*}$ undergoes a simple Hopf bifurcation (with $x_{2}$ as bifurcation parameter).

Proof. It is straightforward to check that the rate constants in Table 1 satisfy the inequality (13). Therefore, the surface $\mathcal{H}$ as in Proposition 4.1.2(b) exists, and is defined by det $H_{5}=0$, where $H_{5}$ is the Hurwitz matrix (specialized to the rate constants in Table 10) as in the proof of Proposition 4.1.

To prove that $\mathcal{H}$ consists generically of Hopf bifurcations, we use Proposition 4.3. That result states that $\chi\left(x_{1}^{*}, x_{2}^{*}, x_{6}^{*}\right)$ is a Hopf bifurcation with respect to $x_{2}$ if and only if $\left(x_{1}^{*}, x_{2}^{*}, x_{6}^{*}\right) \in$ $\mathcal{H}^{\prime} \backslash \mathcal{S}$, where

$$
\begin{aligned}
\mathcal{H}^{\prime} & :=V_{>0}\left(\operatorname{det} H_{5}\right):=\left\{\left(x_{1}, x_{2}, x_{6}\right) \in \mathbb{R}_{>0}^{3} \mid \operatorname{det} H_{5}\left(x_{1}, x_{2}, x_{6}\right)=0\right\}, \text { and } \\
\mathcal{S} & :=\left\{\left(x_{1}^{*}, x_{2}^{*}, x_{6}^{*}\right) \in \mathcal{H}^{\prime}\left|\frac{d\left(\left.\operatorname{det} H_{5}\right|_{x_{1}=x_{1}^{*}, x_{6}=x_{6}^{*}}\right)}{d x_{2}}\right|_{x_{2}=x_{2}^{*}}=0\right\} \subseteq \mathcal{H}^{\prime} .
\end{aligned}
$$

We have that $\mathcal{H}=\phi \circ \chi\left(\mathcal{H}^{\prime}\right)$, and that the following subset of $\mathcal{H}$ consists of compatibility classes whose unique steady state undergoes a simple Hopf bifurcation with $x_{2}$ as bifurcation parameter: $\phi \circ \chi\left(\mathcal{H}^{\prime} \backslash \mathcal{S}\right)$. So, it suffices to show that $\operatorname{dim}(\mathcal{S})<\operatorname{dim}\left(\mathcal{H}^{\prime}\right)$. Note that $\operatorname{dim}\left(\mathcal{H}^{\prime}\right) \geq 2$, so we will show that $\operatorname{dim}(\mathcal{S}) \leq 1$.

To this end, note that if $\left(x_{1}^{*}, x_{2}^{*}, x_{6}^{*}\right) \in \mathcal{S}$, then $x_{2}^{*}$ is a multiple root of the univariate polynomial numerator $\left.\left(\operatorname{det} H_{5}\right)\right|_{x_{1}=x_{1}^{*}, x_{6}=x_{6}^{*}}$ (this also uses the fact the denominator of $\operatorname{det} H_{5}$, which is $188956800000000000000 x_{6}^{5}$, does not depend on $\left.x_{2}\right)$. Thus, any $\left(x_{1}^{*}, x_{2}^{*}, x_{6}^{*}\right) \in \mathcal{S}$ satisfies $D\left(x_{1}^{*}, x_{6}^{*}\right)=0$, where $D$ is the discriminant of det $H_{5}$ and $H_{5}$ is viewed as a univariate polynomial in the variable $x_{2}$. So, we have the map:

$$
\begin{aligned}
\mathcal{S} & \rightarrow\left\{\left(x_{1}, x_{6}\right) \in \mathbb{R}^{2} \mid D\left(x_{1}, x_{6}\right)=0\right\}=: \mathcal{D} \\
\left(x_{1}, x_{2}, x_{6}\right) & \mapsto\left(x_{1}, x_{6}\right) .
\end{aligned}
$$


The preimage of any point of this map has size at most 4 (because numerator $\left.\left(\operatorname{det} H_{5}\right)\right|_{x_{1}=x_{1}^{*}, x_{6}=x_{6}^{*}}$ has degree 9 , so it has at most 4 multiple roots).

Thus, to achieve our desired inequality (namely, $\operatorname{dim}(\mathcal{S}) \leq 1$ ), we need only prove the following claim: $\operatorname{dim}(\mathcal{D}) \leq 1$ or, equivalently, the bivariate polynomial $D$ is not the zero polynomial. It suffices to show that $D(1,1)$ is nonzero, which in turn would follow if we can show that the univariate, degree-9 polynomial numerator $\left.\left(\operatorname{det} H_{5}\right)\right|_{x_{1}=x_{1}^{*}, x_{6}=x_{6}^{*}}=H_{5}\left(1, x_{2}, 1\right)$ does not have a multiple root over $\mathbb{C}$. Indeed, using Mathematica, we see that the numerator of det $H_{5}\left(1, x_{2}, 1\right)$ has 9 (distinct) complex roots:

$-131.425, \quad-102.999, \quad-78.022, \quad-66.423, \quad-39.194, \quad-3.946 \pm 0.734 i, \quad-3.677,268.606$.

Thus, $D$ is a nonzero polynomial, and this completes the proof.

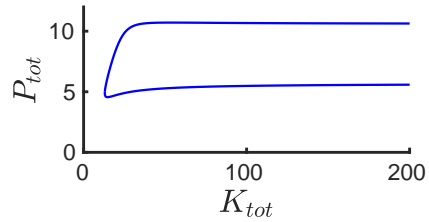

(a) $S_{\text {tot }}=40$.

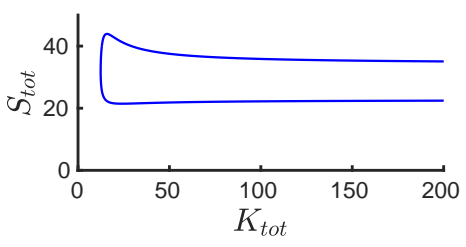

(b) $P_{\text {tot }}=5$.

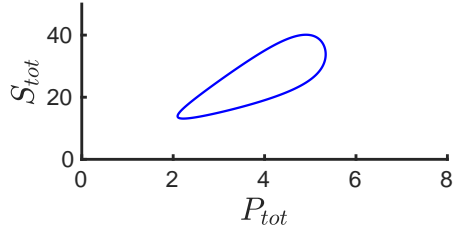

(c) $K_{\text {tot }} \approx 13.0296$.

Figure 3: Slices of the Hopf-bifurcation surface $\mathcal{H}$, from Theorem 4.5, Specifically, displayed are the intersections of $\mathcal{H}$ with the hyperplanes defined by (a) $S_{\text {tot }}=40$, (b) $P_{\text {tot }}=5$, and (c) $K_{\text {tot }} \approx 13.0296$. Each such curve was obtained numerically, using Matcont [8], by a two-parameter continuation of the Hopf bifurcation arising from $K_{\text {tot }} \approx 13.0296, P_{\text {tot }}=5$, and $S_{\text {tot }}=40$. Each point of the curves in $(\mathrm{a})-(\mathrm{c})$ corresponds to a Hopf bifurcation with respect to either of the two varying total concentrations. Points "inside" $\mathcal{H}$ correspond to unstable steady states and thus the potential for oscillations.

In Figure 3, we show some slices of the Hopf-bifurcation surface $\mathcal{H}$ (where the rate constants are from Table 1). Accordingly, this figure extends the one-dimensional Figure 1.

The bifurcations analyzed in Proposition 4.3 and Theorem 4.5 are with respect to the bifurcation parameter $x_{2}$, the steady-state value of the kinase $K$. It is natural to ask whether we also obtain a bifurcation with respect to a more biologically meaningful parameter, such as a rate constant or a total amount. We now explain how to perform such an analysis.

To use a total amount (here we use $P_{\text {tot }}$ ) as a bifurcation parameter (perturbing this parameter corresponds to perturbing the compatibility class), consider the following maps:

$$
\left\{\left(K_{\text {tot }}, P_{\text {tot }}, S_{\text {tot }}\right)\right\}=\mathbb{R}_{>0}^{3} \stackrel{\phi \circ \chi}{\longleftarrow} \mathbb{R}_{>0}^{3} \stackrel{h_{5}:=\operatorname{det} H_{5}}{\longrightarrow} \mathbb{R}_{>0}
$$

Recall that $(\phi \circ \chi): \mathbb{R}_{>0}^{3} \rightarrow \mathbb{R}_{>0}^{3}$ is a bijection. Let $\mathfrak{g}:=h_{5} \circ(\phi \circ \chi)^{-1}: \mathbb{R}_{>0}^{3} \rightarrow \mathbb{R}$. Also, let $p:=(\phi \circ \chi)_{2}=x_{6}+\frac{1009}{1800} x_{1} x_{2}$ denote the second coordinate function of $\phi \circ \chi$ from (9) (here we assume the rate constants from Table 1). We are interested in checking whether $\frac{\partial \mathfrak{g}}{\partial P_{\text {tot }}}$ is 
(generically) nonzero whenever $\mathfrak{g}=0$. Accordingly, we use the chain rule:

$$
\begin{aligned}
\frac{\partial \mathfrak{g}}{\partial P_{\text {tot }}} & =\frac{1}{\partial p / \partial x_{1}} \frac{\partial h_{5}}{\partial x_{1}}+\frac{1}{\partial p / \partial x_{2}} \frac{\partial h_{5}}{\partial x_{2}}+\frac{1}{\partial p / \partial x_{6}} \frac{\partial h_{5}}{\partial x_{6}} \\
& =\frac{1800}{1009 x_{2}} \frac{\partial h_{5}}{\partial x_{1}}+\frac{1800}{1009 x_{1}} \frac{\partial h_{5}}{\partial x_{2}}+\frac{\partial h_{5}}{\partial x_{6}} .
\end{aligned}
$$

For specific values of $x_{1}, x_{2}, x_{6}$, it is straightforward to check whether the sum (17) is nonzero. More generally, we expect this sum to be generically nonzero; that is, we expect that the surface $\mathcal{H}$ consists generically of Hopf bifurcations with respect to the total-amount $P_{\text {tot }}$.

\section{$5 \quad$ Generating rate constants admitting oscillations}

The proof of Theorem 4.2 yields a recipe for generating rate constants for the mixedmechanism network at which we expect oscillations arising from a Hopf bifurcation. Specifically, we choose rate constants $k_{i}$ for which the equalities $k_{2}=k_{6}=k_{9}$ hold, the inequality (13) holds, and $\alpha_{0}<0$ (as in (15)), and then pick $x_{2}$ and $x_{6}$ large enough so that det $H_{5}$ is negative but close to 0 . We summarize these choices in the following procedure.

Procedure 5.1 (Generating rate constants likely to admit oscillations).

Input: The following function 3 :

(i) $\alpha_{0}$ as in (15),

(ii) the numerator of $\operatorname{det} H_{5}$,

(iii) $q:=\alpha_{0} x_{6}^{3}+\alpha_{1} x_{6}^{2}+\alpha_{2} x_{6}+\alpha_{3}$ as in (14), and

(iv) $\phi \circ \chi$ given in Proposition 3.6 .

Output: Rate constants and total amounts for which det $H_{5}$ is negative and close to 0. Steps:

1. Choose positive values for $k_{b}:=k_{2}=k_{6}=k_{9}, x_{1}, k_{1}, k_{3}, k_{4}, k_{7}$, and $k_{8}$.

2. Choose a positive value for $k_{10}$ for which $k_{10}>\frac{\left(k_{3}+k_{4}\right)\left(k_{3}+k_{7}\right)\left(k_{4}+k_{7}\right)}{k_{3} k_{4}}$.

3. Choose the remaining rate constant $k_{5}$ such that $\alpha_{0}<0$.

4. Choose $x_{6}$ so that $q<0$.

5. Choose $x_{2}$ so that the numerator of det $H_{5}$ is negative but close to 0 .

6. Return the $k_{i}$ 's and $\left(K_{\text {tot }}, P_{\text {tot }}, S_{\text {tot }}\right):=\phi \circ \chi\left(x_{1}, x_{2}, x_{6}\right)$, where $\phi \circ \chi$ is evaluated at the $k_{i}$ 's (and $x_{1}, x_{2}, x_{6}$ ) chosen in the previous steps.

Remark 5.2. Using the output of Procedure 5.1, one can attempt to exhibit and analyze oscillations or Hopf bifurcations using software, e.g., Matcont [8]. See Figure 4,

\footnotetext{
${ }^{3}$ The functions are provided as a text file in the Supporting Information. See Appendix A.
} 
Example 5.3. We follow Procedure 5.1 as follows (to verify our computations see the file mixed_generate_rc.nb):

Step 1. We pick $k_{b}=0.143738, k_{1}=0.575284, k_{3}=3.89096, k_{4}=5.05386, k_{7}=9.25029$, $k_{8}=0.621813$, and $x_{1}=5.82148$.

Step 2. The inequality for this step evaluates to $k_{10}>85.5048$, so we choose $k_{10}=90$.

$\overline{\text { Step 3 }}$. Evaluating $\alpha_{0}$ at the chosen $k_{i}$ 's, we obtain the following inequality:

$$
-8.896 \times 10^{17} k_{5}^{3}+1.49735 \times 10^{20} k_{5}^{2}+4.79701 \times 10^{20} k_{5}+2.42695 \times 10^{20}<0,
$$

which we find, using Mathematica, is feasible for $k_{5}>171.471$. So, we pick $k_{5}=172$.

Step 4. By evaluating $q$ at the values chosen above, we obtain the following inequality:

$$
-1.41683 \times 10^{22} x_{6}^{3}-3.5508 \times 10^{25} x_{6}^{2}-1.80374 \times 10^{25} x_{6}+2.15078 \times 10^{24}<0 .
$$

This inequality holds when $x_{6}>0.0996797$, so we choose $x_{6}=0.1$.

Step 5. By evaluating the numerator of det $H_{5}$, we obtain the following inequality:

$$
\begin{aligned}
- & 5.42893 \times 10^{25} x_{2}^{9}-4.20944 \times 10^{29} x_{2}^{8}-5.05393 \times 10^{31} x_{2}^{7}-6.67609 \times 10^{32} x_{2}^{6} \\
& +4.66164 \times 10^{33} x_{2}^{5}+3.97617 \times 10^{34} x_{2}^{4}+1.01289 \times 10^{35} x_{2}^{3}+1.19894 \times 10^{35} x_{2}^{2} \\
& +6.7831 \times 10^{34} x_{2}+1.4718 \times 10^{34}<0 .
\end{aligned}
$$

This inequality is feasible, as computed in Mathematica, for $x_{2}>9.0382$; we pick $x_{2}=10$.

Step 6. We have determined the following rate constants:

\begin{tabular}{|c|c|c|c|c|c|c|c|c|c|}
\hline$k_{1}$ & $k_{2}$ & $k_{3}$ & $k_{4}$ & $k_{5}$ & $k_{6}$ & $k_{7}$ & $k_{8}$ & $k_{9}$ & $k_{10}$ \\
\hline 0.575284 & 0.143738 & 3.89096 & 5.05386 & 172 & 0.143738 & 9.25029 & 0.621813 & 0.143738 & 90 \\
\hline
\end{tabular}

We obtain the following steady state, using (7):

$$
\begin{aligned}
\left(x_{1}, x_{2}, \ldots, x_{9}\right) & =\chi\left(x_{1}, x_{2}, x_{6}\right) \\
& =(5.82148,10,8.30052,6.39056,1.90691,0.1,3.49146,520.229,0.358855)
\end{aligned}
$$

Using this steady state, we obtain the total amounts, using (8):

$$
\left(K_{\text {tot }}, P_{\text {tot }}, S_{\text {tot }}\right)=\phi\left(x_{1}, x_{2}, \ldots, x_{9}\right)=(24.6911,3.95031,546.499) .
$$

The resulting bifurcation analysis is shown in Figure 4.

\section{Dynamics: simulations and conjectures}

Are oscillations the norm when the mixed-mechanism system has an unstable steady state? We conjecture that this is the case.

Conjecture 6.1. Consider the mixed-mechanism network, and any choice of rate constants and total amounts. If the unique steady state in $\mathcal{P}$ is unstable, then $\mathcal{P}$ contains a stable periodic orbit. 


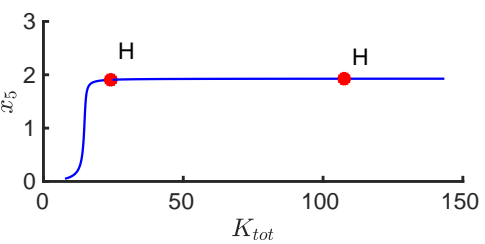

(a) Bif. parameter $K_{\text {tot }}$.

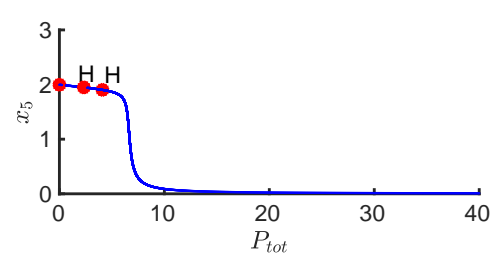

(b) Bif. parameter $P_{\text {tot}}$.

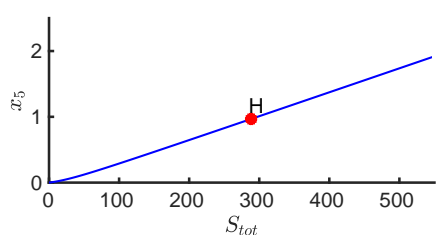

(c) Bif. parameter $S_{\text {tot }}$.

Figure 4: Numerical continuation of the steady state (18), when total amounts are as in (19): (a) A (supercritical) Hopf bifurcations are at $K_{\text {tot }} \approx 24.0623$ and 107.5635. (b) (Supercritical) Hopf bifurcations are at $P_{\text {tot }} \approx 4.1022$ and $P_{\text {tot }} \approx 2.3275$. Matcont reported a branch point, the leftmost red circle, at $P_{t o t} \approx-8.5427 \times 10^{-13}$, i.e., for $P_{\text {tot }} \approx 0$ and thus outside the domain of interest. (c) A (supercritical) Hopf bifurcation is at $S_{\text {tot }} \approx 288.4384$.

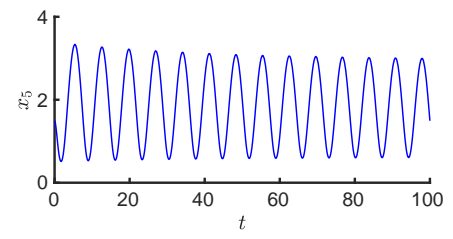

(a) $x_{5}$ vs. $t$.

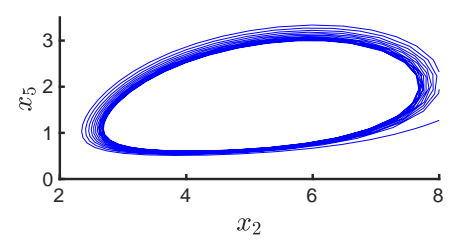

(b) $x_{5}$ vs. $x_{2}$.

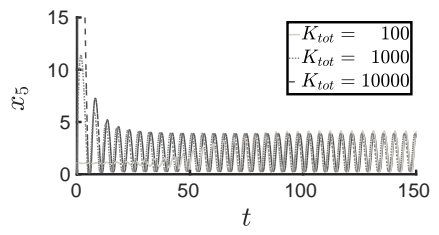

(c) Increasing $K_{t o t}$.

Figure 5: Numerical verification of oscillations in the mixed-mechanism system with rate constants as in Table 1. For (a) and (b), we used $\left(K_{\text {tot }}, P_{\text {tot }}, S_{\text {tot }}\right)=(14,5,40)$ and initial values as in (10). Here the solution converges to a periodic orbit. For (c), we used $\left(P_{\text {tot }}, S_{\text {tot }}\right)=(8,40)$ and three values for $K_{\text {tot }}$ (namely, 100, 1000, and 10000), and again initial values as in (10), except that $x_{5}=1.1$. Again the solutions seem to converge to a periodic orbit, and moreover this periodic orbit appears not to depend on the value of $K_{\text {tot }}$. See Conjecture 6.2.

Some simulations are shown in Figure 5. In (A) and (B) of that figure, we see solutions converging to a period orbit; this system arises from total-amounts similar to those that Suwanmajo and Krishnan found to support oscillations. In contrast, in Figure 5 (C), we see oscillations, when $\left(P_{\text {tot }}, S_{\text {tot }}\right)=(8,40)$, for three large values for $K_{\text {tot }}: 100,1000$, and 10000. Oscillations persist across these values, which yields a much larger range for $K_{\text {tot }}$ than Suwanmajo and Krishnan's results would suggest.

Moreover, the value of $K_{\text {tot }}$ appears not to affect the resulting periodic orbit (when projected to $x_{5}$, the concentration of the doubly phosphorylated substrate $S_{2}$ ). Could this be a biological design mechanism for robust timekeeping (for instance, in circadian clocks)? Mathematically, we conjecture that oscillations indeed persist for arbitrarily large $K_{\text {tot }}$; and, that the periodic orbit in $x_{5}$ indeed does not depend on $K_{\text {tot }}$.

\section{Conjecture 6.2.}

1. Consider the mixed-mechanism network with rate constants as in Table 1. Then there exist values of $P_{\text {tot }}$ and $S_{\text {tot }}$ such that for $K_{\text {tot }}$ arbitrarily large, the unique steady state in $\mathcal{P}$ is unstable. 
2. For such values of $P_{\text {tot }}$ and $S_{\text {tot }}$ and for sufficiently large $K_{\text {tot }}$, the compatibility class $\mathcal{P}$ contains a periodic orbit such that this orbit in $x_{5}$ (the concentration of $S_{2}$ ) does not depend on the value of $K_{\text {tot }}$.

One way to tackle Conjecture 6.2 is to analyze the robustness of the period and the amplitude with respect to $K_{\text {tot }}$ using the theory developed in [3, 24, 23].

Finally, we consider the dynamics in compatibility classes that contain a locally stable steady state. Our simulations suggest that such a steady state is in fact globally stable. Accordingly, we pose the question, Consider the mixed-mechanism network, and any choice of rate constants and total amounts. If the unique steady state $x^{*}$ in $\mathcal{P}$ is locally stable, does it always follow that $x^{*}$ is globally stable? In the Michaelis-Menten limit, this is true [36].

\section{Discussion}

We return to the question, How do oscillations emerge in phosphorylation networks? Concretely, we would like (1) easy-to-check criteria for exactly which phosphorylation networks admits oscillations or Hopf bifurcations, and (2) for those networks that admit oscillations, a better understanding of the "geography of parameter space", that is, a characterization of which rate constants and initial conditions yield oscillations. Both of these problems are still unresolved, and the second problem in particular is very difficult.

Nevertheless, here we made progress on characterizing some of the geography of parameter space for the mixed-mechanism phosphorylation network. Indeed, we found that a single surface defines the boundary between stable and unstable steady states, and this surface consists generically of Hopf bifurcations. Hence, when a steady state switches from stable to unstable, then we expect it to undergo a Hopf bifurcation leading to oscillations. Additionally, we gave a procedure for generating many parameter values leading to oscillations.

We now discuss the significance of our work. At a glance, it might seem that our results are specific to network (11) and rate constants related to those in Table 11. However, the approach is general: for other rate constants (e.g., estimated from data) or other networks (e.g., a version of the ERK network from [37] also has oscillations and a unique steady state), one could apply the same techniques. Therefore, the potential impact is broad.

Going forward, we hope that the novel techniques we used - specifically, using a steadystate parametrization together with a Hopf-bifurcation criterion - will contribute to solving other problems. For instance, we expect that such tools could help solve an important open problem in this area [7], namely, the question of whether oscillations or Hopf bifurcations arise from the fully distributive phosphorylation network.

\section{Acknowledgements}

AS was partially supported by the NSF (DMS-1312473/1513364 and DMS-1752672) and the Simons Foundation (\#521874). AS thanks Alan Rendall and Jonathan Tyler for helpful discussions. CC was partially supported by the Deutsche Forschungsgemeinschaft DFG (DFG-284057449). The authors two referees for their helpful suggestions. 


\section{A Files in the Supporting Information}

The following files can be found at http://www.math.tamu.edu/ annejls/mixed.html:

Text files:

- mixed_H5N_kb.txt $\ldots$ contains $\mathrm{H} 5 \mathrm{~N}$, the numerator of det $H_{5}$ under the assumption $k_{2}=k_{6}=k_{9}=k_{b}$

- mixed_W.txt ...contains a matrix W that defines (3)

- mixed_xt.txt ...contains xt, the parameterization (7)

- mixed_Jx.txt ...contains Jx, the Jacobian evaluated at the parameterization (7)

Mathematica Notebooks:

- mixed_analysis_H5N_x1_LT.nb:

Functionality: This file can be used to obtain numerator $\left(\operatorname{det} H_{5}\right)$ as in (12), in particular to examine the coefficients $\alpha_{01}, \alpha_{10}, \ldots$

Input: the file mixed_H5N_kb.txt

- mixed_analysis_H5N_x2_LT.nb:

Functionality: This file can be used to obtain numerator (det $\left.H_{5}\right)$ as in (14), in particular to examine the coefficients $\alpha_{0}, \ldots, \alpha_{3}$ and $\beta_{0}, \ldots, \beta_{3}$.

Input: the file mixed_H5N_kb.txt

- mixed_coeffs_charpoly.nb:

Functionality: This file can be used to obtain the characteristic polynomial of the Jacobian of the system (2). It contains the Mathematica commands to establish $b_{i}>0$.

Input: the file mixed_Jx.txt

- mixed_Hi.nb:

Functionality: This file can be used to obtain the determinants of the Hurwitz matrices

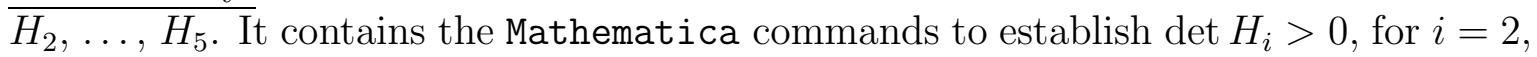
3,4 and that det $H_{5}$ is of mixed sign.

Input: the file mixed_Jx.txt

- mixed_generate_rc.nb:

Functionality: This file contains a realization of Procedure 5.1.

Input: the files mixed_H5N_kb.txt, mixed_W.txt, mixed_xt.txt, mixed_Jx.txt.

\section{References}

[1] Kazuhiro Aoki, Masashi Yamada, Katsuyuki Kunida, Shuhei Yasuda, and Michiyuki Matsuda, Processive phosphorylation of ERK MAP kinase in mammalian cells, P. Natl. Acad. Sci. USA 108 (2011), no. 31, 12675-12680.

[2] Peter Atkins, Julio De Paula, and James Keeler, Atkins' physical chemistry, Oxford University Press, 2018. 
[3] EG Bure and Ye N Rozenvasser, On investigations of autooscillating system sensitivity, Avtomat. i Telemekh (1974), no. 7, 9-17.

[4] Carsten Conradi, Elisenda Feliu, Maya Mincheva, and Carsten Wiuf, Identifying parameter regions for multistationarity, PLoS Comput. Biol. 13 (2017), no. 10, e1005751.

[5] Carsten Conradi and Maya Mincheva, Catalytic constants enable the emergence of bistability in dual phosphorylation, J. R. Soc. Interface 11 (2014), no. 95.

[6] Carsten Conradi and Anne Shiu, A global convergence result for processive multisite phosphorylation systems, B. Math. Biol. 77 (2015), no. 1, 126-155. MR 3303108

[7] _ Dynamics of post-translational modification systems: recent progress and future challenges, Biophys. J. 114 (2018), no. 3, 507-515.

[8] Annick Dhooge, Willy Govaerts, and Yuri A. Kuznetsov, MATCONT: A MATLAB package for numerical bifurcation analysis of ODEs, ACM Trans. Math. Softw. 29 (2003), no. 2, 141-164.

[9] Mirela Domijan and Markus Kirkilionis, Bistability and oscillations in chemical reaction networks, J. Math. Biol. 59 (2009), no. 4, 467-501.

[10] Mitchell Eithun and Anne Shiu, An all-encompassing global convergence result for processive multisite phosphorylation systems, Math. Biosci. 291 (2017), 1-9.

[11] Hassan Errami, Markus Eiswirth, Dima Grigoriev, Werner M. Seiler, Thomas Sturm, and Andreas Weber, Detection of Hopf bifurcations in chemical reaction networks using convex coordinates, J. Comput. Phys. 291 (2015), 279-302.

[12] James E Ferrell and Sang Hoon Ha, Ultrasensitivity part II: multisite phosphorylation, stoichiometric inhibitors, and positive feedback, Trends Biochem. Sci. 39 (2014), no. 11, $556-569$.

[13] Feliks R. Gantmacher, Matrix theory, Chelsea, New York 21 (1959).

[14] Karin Gatermann, Markus Eiswirth, and Anke Sensse, Toric ideals and graph theory to analyze Hopf bifurcations in mass action systems, J. Symbolic Comput. 40 (2005), no. $6,1361-1382$.

[15] I.M. Gelfand, M.M. Kapranov, and A.V. Zelevinsky, Discriminants, resultants and multidimensional determinants, Birkhäuser, 1994.

[16] John Guckenheimer and Philip Holmes, Nonlinear oscillations, dynamical systems, and bifurcations of vector fields, vol. 42, Springer Science \& Business Media, 2013.

[17] Jeremy Gunawardena, Multisite protein phosphorylation makes a good threshold but can be a poor switch, P. Natl. Acad. Sci. USA 102 (2005), no. 41, 14617-14622. 
[18] Otto Hadač, František Muzika, Vladislav Nevoral, Michal Přibyl, and Igor Schreiber, Minimal oscillating subnetwork in the Huang-Ferrell model of the MAPK cascade, PLOS ONE 12 (2017), no. 6, 1-25.

[19] Juliette Hell and Alan D. Rendall, A proof of bistability for the dual futile cycle, Nonlinear Anal.-Real 24 (2015), 175-189.

[20] Zoe Hilioti, Walid Sabbagh, Saurabh Paliwal, Adriel Bergmann, Marcus D Goncalves, Lee Bardwell, and Andre Levchenko, Oscillatory phosphorylation of yeast Fus3 MAP kinase controls periodic gene expression and morphogenesis, Curr. Biol. 18 (2008), no. 21, 1700-1706.

[21] Huizhong Hu, Alexey Goltsov, James L Bown, Andrew H Sims, Simon P Langdon, David J Harrison, and Dana Faratian, Feedforward and feedback regulation of the MAPK and PI3K oscillatory circuit in breast cancer, Cell. Signal. 25 (2013), no. 1, 26-32.

[22] Wolfram Research, Inc., Mathematica, Version 11.3, Champaign, IL, 2018.

[23] Brian Ingalls, Maya Mincheva, and Marc R. Roussel, Parametric sensitivity analysis of oscillatory delay systems with an application to gene regulation, B. Math. Biol. 79 (2017), no. 7, 1539-1563.

[24] Brian P Ingalls, Autonomously oscillating biochemical systems: parametric sensitivity of extrema and period, Systems biol. 1 (2004), no. 1, 62-70.

[25] Matthew D. Johnston, Translated chemical reaction networks, B. Math. Biol. 76 (2014), no. $6,1081-1116$.

[26] Matthew D. Johnston, Stefan Müller, and Casian Pantea, A deficiency-based approach to parametrizing positive equilibria of biochemical reaction systems, Preprint, arXiv:1805.09295 (2018).

[27] Wei Min Liu, Criterion of Hopf bifurcations without using eigenvalues, J. Math. Anal. Appl. 182 (1994), no. 1, 250-256. MR 1265895

[28] German Lozada-Cruz, The simple application of the implicit function theorem, Boletin de la Asociatión Matemática Venezolana XIX (2012), no. 1.

[29] Stefan Müller, Elisenda Feliu, Georg Regensburger, Carsten Conradi, Anne Shiu, and Alicia Dickenstein, Sign conditions for injectivity of generalized polynomial maps with applications to chemical reaction networks and real algebraic geometry, Found. Comput. Math. 16 (2016), no. 1, 69-97.

[30] Koji L. Ode and Hiroki R. Ueda, Design principles of phosphorylation-dependent timekeeping in eukaryotic circadian clocks, Cold Spring Harbor Perspectives in Biology (2017).

[31] Parag Patwardhan and W. Todd Miller, Processive phosphorylation: Mechanism and biological importance, Cell. Signal. 19 (2007), no. 11, 2218-2226. 
[32] Mercedes Pérez Millán and Alicia Dickenstein, The structure of MESSI biological systems, SIAM J. Appl. Dyn. Syst. 17 (2018), no. 2, 1650-1682.

[33] Mercedes Pérez Millán, Alicia Dickenstein, Anne Shiu, and Carsten Conradi, Chemical reaction systems with toric steady states, B. Math. Biol. 74 (2012), no. 5, 1027-1065.

[34] Mercedes Pérez Millán and Adrián G. Turjanski, MAPK's networks and their capacity for multistationarity due to toric steady states, Math. Biosci. 262 (2015), 125-137.

[35] Shodhan Rao, Global stability of a class of futile cycles, J. Math. Biol. 74 (2017), 709726.

[36] _ Stability analysis of the Michaelis-Menten approximation of a mixed mechanism of a phosphorylation system, Math. Biosci. 301 (2018), 159 -166.

[37] Boris Y. Rubinstein, Henry H. Mattingly, Alexander M. Berezhkovskii, and Stanislav Y. Shvartsman, Long-term dynamics of multisite phosphorylation, Mol. Biol. Cell 27 (2016), no. 14, 2331-2340.

[38] Carlos Salazar and Thomas Höfer, Multisite protein phosphorylation - from molecular mechanisms to kinetic models, FEBS Journal 276 (2009), no. 12, 3177-3198.

[39] Thapanar Suwanmajo and J. Krishnan, Mixed mechanisms of multi-site phosphorylation, J. R. Soc. Interface 12 (2015), no. 107.

[40] Matthew Thomson and Jeremy Gunawardena, The rational parameterisation theorem for multisite post-translational modification systems, J. Theoret. Biol. 261 (2009), no. 4, 626-636.

[41] Hwai-Ray Tung, Precluding oscillations in Michaelis-Menten approximations of dualsite phosphorylation systems, Preprint, arXiv:1712.03594 (2017).

[42] David M. Virshup and Daniel B. Forger, Keeping the beat in the rising heat, Cell 137 (2009), no. 4, 602-604.

[43] Xiaojing Yang, Generalized form of Hurwitz-Routh criterion and Hopf bifurcation of higher order, Appl. Math. Lett. 15 (2002), no. 5, 615-621. 